\title{
Editorial
}

\section{Criação da ABECiP}

Foi criada oficialmente a Associação Brasileira de Editores Científicos em Psicologia (ABECiP), com o apoio da biblioteca Virtual de Psicologia (BVS-Psi) e da Associação Nacional de Pesquisa e Pós-graduação em Psicologia (ANPEPP). A apresentação do estatuto e a aclamação da Diretoria ocorreram durante o II Encontro de Editores de Revistas Científicas da Área de Psicologia, realizado em São Paulo, no dia 10 de março de 2006.

Além da criação da ABECiP, o Encontro foi oportuno para tratar de importantes questões para o futuro dos periódicos da área. André Serradas apresentou o PePSIC, portal eletrônico de periódicos de revistas de Psicologia e áreas afins, fruto da parceria entre a BVS-Psi e a ABECiP. O PEPSic contou com o apoio do Centro Latino Americano e do Caribe de Informação em Ciências da Saúde (BIREME), que cedeu a metodologia - Scientific Electronic Library Online (SciELO) para publicação de periódicos em formato eletrônico e sua disponibilização gratuita na Internet. A proposta de criação de uma cooperativa de revistas interessadas na publicação eletrônica no PePSIC foi bem vinda pelos editores como uma forma de agilizar a publicação e reduzir custos.

O Sistema SciELO de Publicação/OJS foi apresentado por Adriana Luccisano. Esse Sistema, em fase de testes, é um gerenciador do processo editorial da revista, que ao final do processo gera um artigo pronto para a publicação na SciELO. A metodologia é semelhante ao já usado pela Psicologia Reflexão e Crítica (Sistema Eletrônico de Editoração de Revistas - SEER, software distribuído pelo IBICT), pois no final do processo editorial é gerado um arquivo para disponibilização eletrônica.

Aparecida Angélica Sabadini apresentou a estrutura do Manual dos Editores e Autores Psi, que será um guia para editores científicos da área, muito esperado entre os editores e certamente entre autores.

A questão da avaliação de revistas e da qualidade dos periódicos foi amplamente discutida após a apresentação de Piotr Trzesniak, que salientou a importância da editoração eletrônica e a disponibilização eletrônica gratuita através do SciELO e PePSIC. Ele estimulou que pesquisadores representativos da área publiquem em revistas que utilizem SEER, SCIELO ou PePSIC para disponibilizar mais trabalhos em revistas de acesso livre. Como incentivo ao acesso livre, é importante também aumentar o número de citações de revistas de acesso livre e participação em Associação que publique ou assine revistas de acesso livre. Finalmente, Marco Aurelio Maximo Prado informou o programa de trabalho da Subcomissão de Avaliação de Periódicos que apresentou uma proposta de avaliação de periódicos para ser discutida por editores através do site da ANPEPP.

A ABECiP foi uma iniciativa surgida em agosto de 2004, no I Encontro de Editores de Revistas Científicas da Área de Psicologia, quando o PePSIC e o Manual dos Editores e Autores Psi foram projetados. Os membros desta primeira diretoria da ABECiP são: Sebastião Rogério Góis Moreira, da Fundação Helena Antipoff (Presidente), Fermino Fernandes Sisto, da Universidade de São Francisco (Presidente Futuro), Aidyl Macedo de Queiroz Perez-Ramos, da Academia Paulista de Psicologia (Presidente Passado), Maria Inês Gandolfo Conceição, da Universidade de Brasília (1 ${ }^{a}$ Secretária), Maria Imaculada Cardoso Sampaio, da Universidade de São Paulo (2 ${ }^{\text {a }}$ Secretária), Lisiane Bizarro Araújo, da Universidade Federal do Rio Grande do Sul (1 ${ }^{\text {a }}$ Tesoureira) e Piotr Trzesniak, da Universidade Federal de Itajubá $\left(2^{\circ}\right.$ Tesoureiro). O Conselho Fiscal tem como Titulares Ana Maria Jacó Vilela, da Universidade do Estado do Rio de Janeiro, Fuad Kyrillos Neto, da Universidade Presidente Antonio Carlos, Rozilda das Neves Alves, da Universidade Estadual de Maringá, e Fabián Rueda, da Universidade São Francisco; e os Suplentes são Alexandre Dittrich, da Universidade Federal do Paraná e Zália Maria Mendes Biasoli Alves, da Universidade de São Paulo. As ações da ABECiP continuarão representando o interesse da área em consolidar a divulgação da produção científica no Brasil.

Lisiane Bizzaro

Editora Geral 